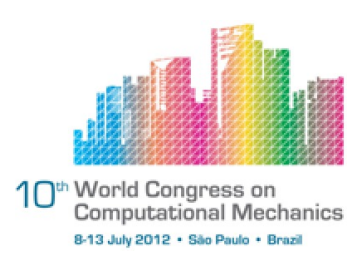

\title{
LIMIT ANALYSIS ON SCRATCH TEST PROBLEM
}

\author{
F. C. Figueiredo ${ }^{1,2}$, L. M. S. A. Borges ${ }^{2}$ \\ ${ }^{1}$ Department of Mechanical Engineering, CEFET/RJ (ffigueiredo@mecsol.ufrj.br) \\ ${ }^{2}$ Department of Mechanical Engineering, Federal University of Rio de Janeiro
}

\begin{abstract}
Scratch test is one of the oldest method to determine mechanical properties in materials. The technique has gained interest due to the properties implied during test, such as adherence, hardness, cohesion and elasticity. On this test a rigid indenter is dragged on the material test surface at a constant depth and controlled forces. This problem is modeled by finite elements method and limit analysis theory, which is is a direct method and there is no need to calculate stresses during each load step in order to compute critical states and collapse mechanisms. From virtual power principle, the internal power is related to the external power, which is amplified by load factor so that the body achieves a plastic collapse. The solution of a limit analysis problem is to find this load factor, the plastically admissible stresses fields that are in equilibrium with the given forces system, the kinematically admissible velocity fields, the strain rate field, which is related to the velocity field by means of a deformation operator, and the plastic multiplier. The solution of a limit analysis problem closely depends on the yield function that describes the behavior of a material, such as von Mises for metals and Drucker-Prager for rocks. However, traditional criteria for rocks and soils such as MohrCoulomb and Drucker-Prager do not consider material porosity. Therefore, an yield criterion that includes porosity effect is applied in this work. This function is applied to the discretized limit analysis model. A semi-analytical solution based on limit analysis lower bound method is developed and the proposed yield function is also applied. Then, the results between both methods are compared.
\end{abstract}

Keywords: Limit Analysis, Finite Elements, Porous Materials, Lower-Bound Solution.

\section{INTRODUCTION}

Scratch tests are among the oldest method to determine mechanical properties, such as adhesion of coatings or strength of materials. On these tests a rigid indenter at a constant depth is dragged on the material test surface. This problem can be modeled under plasticity theory, since a plastic flow occurs during dragging process. The development of plasticity studies on porous materials have wide applications like the determination of material hardness using nondestructive methods like scratch tests. Scratch tests are also used as an alternative way of 
measuring mechanical properties as adhesion of coatings or strength of rocks, according to [1].

Modeling the mechanical behavior of porous materials is a difficult task because of the many variables involved. Porous materials comprise soils (sand, clay), ceramics or even metallic powder, where the metal is physically divided into many small particles, then passing through compression and sintering process. Unlike metals, porous materials are very heterogeneous, composed by many particles, with different sizes, inclusions and of course, porous. The challenge is to quantify the interaction of this many variables into a yield function, important to predict the plastic behavior of the material. The works of [2] and [3] have given a valuable contribution in the development of an yield criterion. This formulation is derived from a micro or nano scale study of solid and porous phases of the materials. Then, an expression for the yield criterion on a macro scale is derived from homogenization theory, expressing solid phase properties as well as information of porous morphology. Variables such as friction angle, cohesion and porosity are take into account in order to determine the material properties.

Based on limit analysis theory and finite element method the scratch test problem is studied and the incipient plastic collapse factors is related to the micro or nano hardness of the porous material[]. The called Ulm-Gathier yield function is applied into the model and some discussions about its Hessian is made. It should be remarked that under certain conditions, this function tends asymptotically to von Mises or Drucker-Prager criteria. As plane strain hypothesis is applied into model, the Ulm-Gathier is developed concerning this hypothesis.

Then, a model validation is made when Ulm-Gathier tends asymptotically to a DruckerPrager criterion, reached when porosity tends to null or, equivalently, packing density tending to $100 \%$. After validating, a solution considering porosity is made. Moreover, a lower-bound semi-analytical solution is developed and used to compare to finite element one.

\section{MODEL}

Schematically, Figure 1 describes the geometry of the scratch test problem:

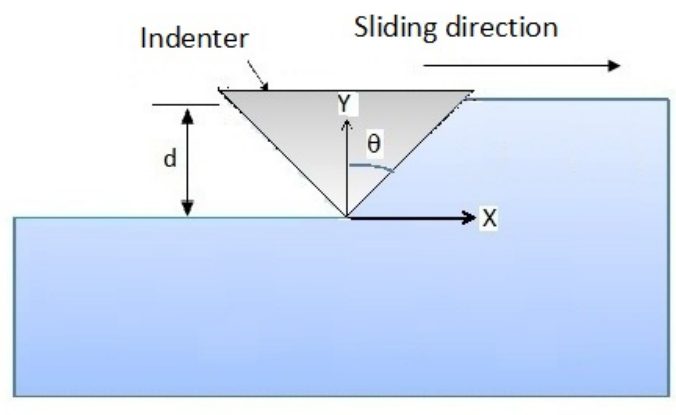

Figure 1. Geometry of the scratch test problem.

In order to model this problem, some basic hypothesis are taken: the contact between indenter and material is frictionless and plane strain is considered.

There are two methods to solve problems in plasticity: the incremental one, which there is need to calculate stress at each load step and the called direct method, represented by 
limit analysis method. This method will be applied in this problem and it is shown a sharp interface with mathematical programming and an optimization problem with restrains. The Finite Element method is also applied.

\subsection{Limit Analysis Model}

Limit analysis method aims the determination of the loads that will cause the phenomenon of incipient plastic collapse, in a body made of elastic ideally plastic material as seen in [4] and [5]. The basic concepts of limit analysis are found in [6], [7], [8], [9], among others. The extremum principles for limit analysis of continuum bodies under proportional loads are presented hereafter.

Considering a body that occupies a region $\mathcal{B}$ with regular boundary $\Gamma$ and let $\mathrm{V}$ the function space of all admissible velocity fields $v$ complying with homogeneous boundary conditions prescribed on $\Gamma_{u}$ of $\Gamma$. The strain rate tensor denoted by $\mathrm{D}$ relates with $v$ by a linear operator and the duality product between stress fields $\mathrm{T}$ and strain rate $\mathrm{D}$ belonging respectively from spaces $W^{\prime}$ and $\mathrm{W}$ is written as:

$$
\langle T, D\rangle=\int_{\mathcal{B}} T \cdot D d \mathcal{B}
$$

The load system is represented by an element $\mathrm{F}$ from space $V^{\prime}$, dual of $\mathrm{V}$. The duality product is denoted as:

$$
\langle F, v\rangle=\int_{\mathcal{B}} b . v d \mathcal{B}+\int_{\Gamma_{t}} \tau . v d \Gamma
$$

where $b$ and $\tau$ are body and surface forces respectively, $\Gamma_{t}$ is a part of boundary $\Gamma$ where external loads are prescribed.

From equilibrium requirements:

$$
\langle T, D\rangle=\langle F, v\rangle
$$

The stress field $T$ is constrained to fulfill the plastic admissibility condition, belonging to the set $\mathrm{P}$ defined as:

$$
P=\left\{T \in W^{\prime} \mid f(T) \leq 0\right\}
$$

The constitutive relations are derived from principle of maximum dissipation, associating the stress $T$ and plastic dissipation $X\left(D^{p}\right)$ to a given strain rate $D^{p}$, as in Eq. (5):

$$
X\left(D^{p}\right)=\sup _{T^{*} \in P}\left\langle T^{*}, D^{p}\right\rangle
$$

Stress and plastic strain rates are related by an associative flow law and the complementary condition is also used, as seen in [8]. Solving a problem using the limit analysis consists in finding a load factor $\alpha$ such the body undergoes to plastic collapse when subject to a reference load amplified by $\alpha$. From classical extremum principles of limit analysis, the called static, kinematic and mixed formulations are derived and more information about these principles, limit analysis discretized forms and the algorithm that solves the optimization problem are found in [4] and [5]. 


\subsection{Discretization Scheme}

The continuum form of the limit analysis problem is discretized into 2-D mixed finite elements, applied to solve the scratch problem. Triangular elements as in Figure 2 are used, with quadratic interpolation for velocity and linear interpolation for stresses fields, developed by Solid Mechanics Group in COPPE [10]. An adaptive mesh refinement is applied and the main goal of this approach is to achieve a mesh-adaptive strategy accounting for mesh size refinement, as well as redefinition of the element stretching. More details about adaptive approach are in [11] and it allows the choice of mesh refinement level. The initial refinement mesh is uniform and after an adaptive mesh strategy [4] a refined mesh, able to capture the slip-lines, is produced .

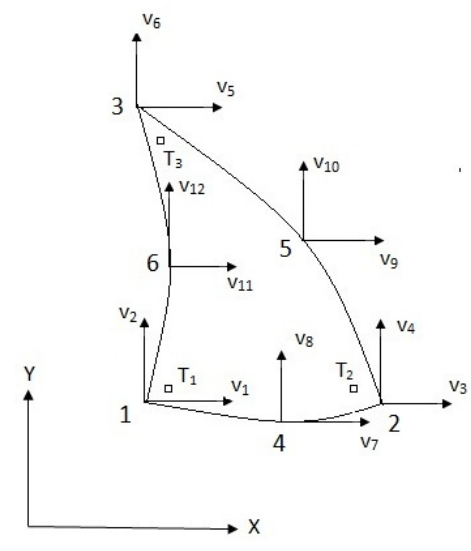

Figure 2. Triangular mixed element.

\section{THE YIELD FUNCTION}

\subsection{Porous materials}

As it is well known, soils are composed by a mix of lots of different particles, creating a very heterogeneous material. Because of its heterogeneity and presence of porous, the determination of mechanical properties of such materials becomes a difficult task. In order to solve this, the development of a predictive model to determine the strength of porous materials will make extensive use of the theory of strength homogenization. Recent advances on homogenization techniques are found in [2].

Porous materials with a dominating matrix-pore inclusion morphology are well represented by the Mori-Tanaka and Self-Consistent schemes, as seen in [2] and [3]. In MoriTanaka scheme some material parameters such as $\alpha_{d}, \sigma_{0}, \alpha_{m}$ are calculated and they include the soil cohesion, porosity and friction angle effects. The called Ulm-Gathier yield function is written in Equation (6):

$$
F\left(\Sigma_{\mathbf{d}}, \Sigma_{m}\right)=\left(\frac{\Sigma_{m}+\sigma_{0}}{\alpha_{m}}\right)^{2}+\left(\frac{J_{2}}{\alpha_{d}}\right)^{2}-1
$$

where $\alpha_{d}, \sigma_{0}, \alpha_{m}$ are material parameters and calculated as seen in [2] and [3], $J_{2}$ is associated to deviatory stress and $\Sigma_{m}$ is mean stress. 
The Ulm-Gathier yield function has an elliptical shape, but using Mori-Tanaka morphology it may assume an hyperbolic shape either, depending on density packing value. Density packing $\eta$ is defined according to Eq. (7):

$$
\eta=\frac{V_{s}}{V_{t}}
$$

where $V_{s}$ is the solid volume and $V_{t}$ is the material total volume, including pores.

Only Mori-Tanaka morphology is applied in this work and according to it, there is a density packing critical value that defines two distinct regimes: below the critical valeu, the yield function assumes an elliptical shape; otherwise, it assumes an hyperbolical shape. This critical density packing $\eta_{\text {crit }}$ is function of friction angle $\alpha_{s}$ and calculated as in Eq. (8):

$$
\eta_{\text {crit }}=1-\frac{4 \alpha_{s}^{2}}{3}
$$

Moreover, under certain special conditions, the Ulm-Gathier function may reach asymptotically either to von Mises or Drucker-Prager criteria: von Mises criterion is obtained if friction angle $\alpha_{s} \rightarrow 0$ and packing density $\eta \rightarrow 1$; however, if $\alpha_{s} \neq 0$ and $\eta=1$, Drucker-Prager criterion is obtained.

Figure 3 shows an example of Ulm-Gathier function, plotting $J_{2}$ (deviatory) versus $\sigma_{m}$ (mean stress) for a friction angle $\alpha_{s}=0.4$. In this case, $\eta_{\text {crit }}=0.786$ and it means that any packing density below this critical value, the yield surface has an elliptical shape and otherwise, the criterion is hyperbolical. It is also observed that when $\eta \rightarrow 1$ the cone-shaped Drucker-Prager criterion is reached. If von Mises citerion were represented, it would be an horizontal line parallel to mean stress axis since Mises is independent of the mean stress, as expected.

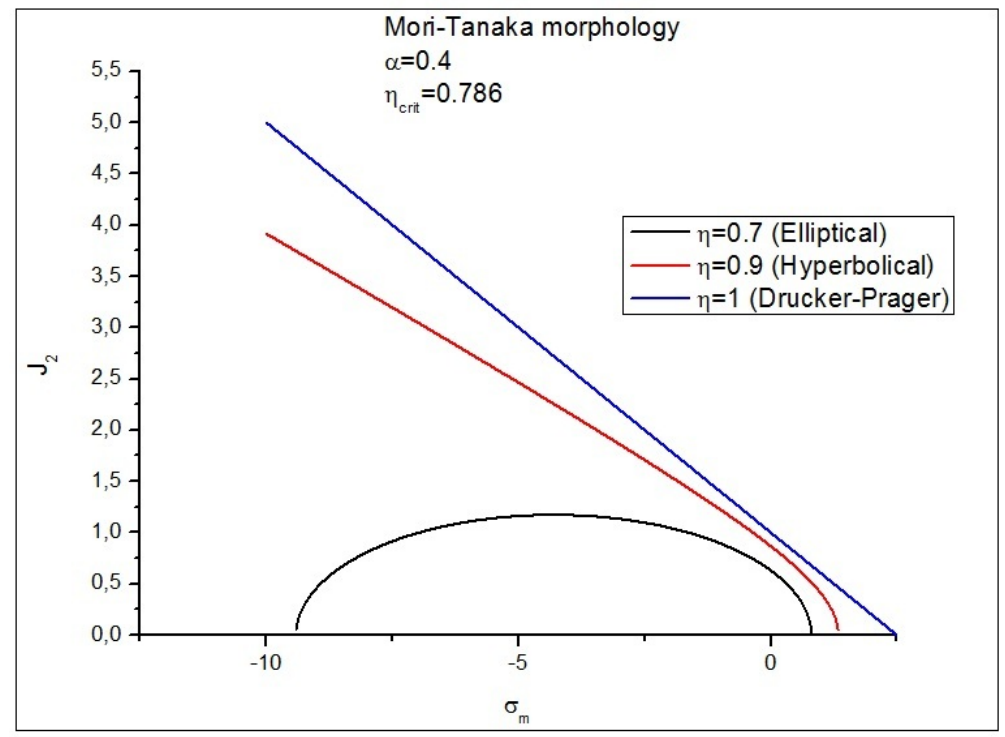

Figure 3. Yield function regimes: elliptical below $\eta_{\text {crit }}$, hyperbolical above this value and limited by Drucker-Prager when $\eta=1$. 


\subsection{The yielding function under plane strain hypothesis}

Denoting $\Sigma$ as the stress field, the deviatory stress is calculated using projection operators, according to Equation (9):

$$
\Sigma_{\mathbf{d}}=\mathbb{P} \Sigma
$$

where tensor $\mathbb{P}$ projects the stress vector $\Sigma$ on deviatory space.

Letting the vector $\mathbf{m}$ representing the unitary vector along hydrostatic direction, the mean stress is calculated as in Equation (10):

$$
\Sigma_{m}=\frac{1}{\sqrt{3}} \Sigma \cdot \mathbf{m}
$$

The decompositions of these stresses components are schematically presented in terms of principal stresses in Figure 4, showing the hydrostatic axis $\mathbf{m}$ and the deviatory plane perpendicular to it:

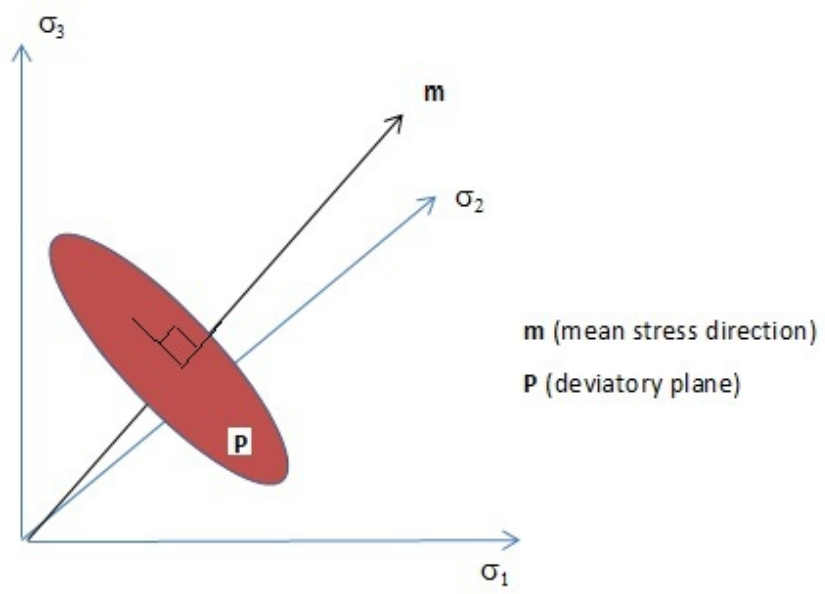

Figure 4. Hydrostatic axis and deviatory plane on principal stresses.

The invariant $J_{2}$ is defined in Eq. (11):

$$
J_{2}=\sqrt{\frac{1}{2} \Sigma_{\mathbf{d}} \cdot \Sigma_{\mathbf{d}}}
$$

From plane deformation hypothesis, the deformation component $\epsilon_{z}$ is made null. Applying the normality rule, then the stress component $\Sigma_{z}$ is obtained in function of $\Sigma_{x}$ and $\Sigma_{y}$ components, as in Equation (12):

$$
\Sigma_{z}=\frac{\Sigma_{x}+\Sigma_{y}}{2} A-B
$$

where: $\mathrm{A}=\frac{\alpha_{m}^{2}-4 \alpha_{d}^{2}}{2 \alpha_{d}^{2}+\alpha_{m}^{2}}$ and $\mathrm{B}=\frac{6 \alpha_{d}^{2} \sigma_{0}}{2 \alpha_{d}^{2}+\alpha_{m}^{2}}$. 
In this way, a relation between a stress vector $\Sigma=\left[\Sigma_{x}, \Sigma_{y}, \Sigma_{z}, \sqrt{2} \Sigma_{x y},\right]^{T}$ and the stress vector at plane deformation denoted by $\Sigma_{\mathbf{p}}$, with components $\left[\Sigma_{x}, \Sigma_{y}, \sqrt{2} \Sigma_{x y}\right]^{T}$ is made through Equation (14):

$$
\Sigma=\mathbb{P}_{D} \Sigma_{\mathbf{p}}+\mathbf{D}
$$

where $\mathbf{D}=[0,0,-B, 0]^{T}$ and $\mathbb{P}_{D}$ is a $4 \times 3$ tensor, defined as follows:

$$
P_{D}=\left[\begin{array}{ccc}
1 & 0 & 0 \\
0 & 1 & 0 \\
A / 2 & A / 2 & 0 \\
0 & 0 & 1
\end{array}\right]
$$

In an alternative way, the yield function described in Equation (6) is rewritten as:

$$
F(\boldsymbol{\Sigma})=\frac{1}{2} \mathbb{C} \boldsymbol{\Sigma} \cdot \boldsymbol{\Sigma}+a \boldsymbol{\Sigma} \cdot \mathbf{m}-r_{k}
$$

where $\mathbb{C}=\frac{1}{\alpha_{d}^{2}} \mathbb{P}+\frac{2}{3 \alpha_{m}^{2}}(\mathbf{m} \otimes \mathbf{m}), a=\frac{2 \sigma_{0}}{\sqrt{3} \alpha_{m}^{2}}$ and $r_{k}=1-\left(\frac{\sigma_{0}}{\alpha_{m}}\right)^{2}$

Rewriting this function using plane deformation formulation:

$$
F\left(\boldsymbol{\Sigma}_{\mathbf{p}}\right)=\frac{1}{2} \mathbb{C}_{p} \boldsymbol{\Sigma}_{\mathbf{p}} \cdot \boldsymbol{\Sigma}_{\mathbf{p}}+\bar{a} \boldsymbol{\Sigma}_{\mathbf{p}} \cdot \mathbf{m}_{\mathbf{p}}+R_{k}
$$

where:

$$
\begin{array}{cc}
\mathbb{C}_{p}=\mathbb{P}_{D}^{T} \mathbb{C} \mathbb{P}_{D} & \mathbf{m}_{\mathbf{p}}=\left[\frac{1}{\sqrt{2}} ; \frac{1}{\sqrt{2}} ; 0\right]^{T} \\
\bar{a}=\frac{3 \sqrt{2} \sigma_{0}}{\left(\alpha_{d}^{2}+3 \alpha_{m}^{2}\right)} & R_{k}=-1+\frac{3 \sigma_{0}^{2}}{\left(\alpha_{d}^{2}+3 \alpha_{m}^{2}\right)}
\end{array}
$$

Once defined the yield function on plane deformation, there is the need to calculate the gradient and the Hessian of Equation (15), which will be implemented in the limit analysis algorithm solver. They are calculated as follows:

$$
\begin{gathered}
\nabla_{\Sigma} F(\Sigma)=\mathbb{C}_{p} \Sigma_{\mathbf{p}}+\bar{a} \mathbf{m}_{\mathbf{p}} \\
\nabla_{\Sigma}^{2} F(\Sigma)=\mathbb{C}_{p}
\end{gathered}
$$

Both gradient and Hessian are used in the limit analysis algorithm and Hessian shall be invertible. In the following the eigenvalues and the eigenvectors of $\mathbb{C}_{p}$ are analyzed. This analysis is important to verify the positive-definiteness of the Hessian, which is convenient to implement in the quasi-Newton limit analysis algorithm.

- Eigenvalues: $\left(\frac{1}{\alpha_{d}^{2}}, \frac{1}{\alpha_{d}^{2}}, \frac{12 \alpha_{d}^{2}+\alpha_{m}^{2}}{\left(2 \alpha_{d}^{2}+\alpha_{m}^{2}\right)^{2}}\right)$

- Eigenvectors: $[0,0,1]^{T},[-1,1,0]^{T},[1,1,0]^{T}$ 
Decomposing $\mathbb{C}_{p}$ using spectral theorem, the characteristic spaces are the deviatory plane and the hydrostatic axis on plane strain, defined by eigenvector $[1,1,0]^{T}$. Thus, every vector on deviatory plane is an eigenvector of $\mathbb{C}_{p}$. Moreover, the positive-definiteness of $\mathbb{C}_{p}$ is proved on elliptical cases since the properties $\alpha_{d}$ and $\alpha_{m}$ are always positive and consequently, the eigenvalues are always positive also.

When the density packing exceeds the critical value, the propriety $\alpha_{d}^{2}$ becomes a negative one. So, doing $\alpha_{d}^{2}=-\left|\alpha_{d}^{2}\right|$ and substituting in Equation (6), it represents an hyperbolic equation and the Hessian in this case would be negative-definite, which is not convenient to implement in the algorithm. Nevertheless, this difficulty can be avoided by adopting a radical function to describe the hyperbolic functions

That is, defining now the tensor $\overline{\mathbb{P}}$ which projects the stress vector $\boldsymbol{\Sigma}_{\mathbf{p}}$ on the deviatory space for plane strain stresses, the yield function on hyperbolical case is determined as follows in Equation:

$$
f\left(\boldsymbol{\Sigma}_{\mathbf{p}}\right)=\bar{J}_{2}-\sqrt{\bar{I}_{1}^{2}}
$$

where:

$$
\begin{gathered}
\bar{J}_{2}=\sqrt{\frac{1}{2} \overline{\mathbb{P}} \Sigma_{\mathbf{p}} \cdot \Sigma_{\mathbf{p}}} \\
I_{1}^{2}=c_{1}\left(\mathbf{m}_{\mathbf{p}} \otimes \mathbf{m}_{\mathbf{p}}\right) \boldsymbol{\Sigma}_{\mathbf{p}} \cdot \boldsymbol{\Sigma}_{\mathbf{p}}+c_{2} \boldsymbol{\Sigma}_{\mathbf{p}} \cdot \mathbf{m}_{\mathbf{p}}-R_{k}
\end{gathered}
$$

The constants $c_{1}, c_{2}$ e $R_{k}$ are calculated as in Equations (23) e (24) :

$$
\begin{gathered}
c_{1}=\frac{-3\left|\alpha_{d}^{2}\right|}{2\left|\alpha_{d}^{2}\right|-6 \alpha_{m}^{2}} \quad c_{2}=2 \sqrt{2} c_{1} \sigma_{0} \\
R_{k}=\left|\alpha_{d}^{2}\right|\left(1+\frac{3 \sigma_{0}^{2}}{\left|\alpha_{d}^{2}\right|-3 \alpha_{m}^{2}}\right)
\end{gathered}
$$

The Gradient and the Hessian of the yield function defined in Equation (20) are as follows in Equations (25) and (26), respectively:

$$
\begin{aligned}
& \nabla f\left(\boldsymbol{\Sigma}_{\mathbf{p}}\right)=\frac{\overline{\mathbb{P}} \boldsymbol{\Sigma}_{\mathbf{p}}}{\sqrt{2 \overline{\mathbb{P}} \boldsymbol{\Sigma}_{\mathbf{p}} \cdot \boldsymbol{\Sigma}_{\mathbf{p}}}}-\frac{2 c_{1}\left(\mathbf{m}_{\mathbf{p}} \otimes \mathbf{m}_{\mathbf{p}}\right) \boldsymbol{\Sigma}_{\mathbf{p}}+c_{2} \mathbf{m}_{\mathbf{p}}}{2 \sqrt{c_{1}\left(\mathbf{m}_{\mathbf{p}} \otimes \mathbf{m}_{\mathbf{p}}\right) \boldsymbol{\Sigma}_{\mathbf{p}} \cdot \boldsymbol{\Sigma}_{\mathbf{p}}+c_{2} \boldsymbol{\Sigma}_{\mathbf{p}} \cdot \mathbf{m}_{\mathbf{p}}-R_{k}}} \\
& \nabla^{2} f\left(\boldsymbol{\Sigma}_{\mathbf{p}}\right)=\frac{1}{\sqrt{2 \overline{\mathbb{P}} \Sigma_{\mathbf{p}} \cdot \Sigma_{\mathbf{p}}}}\left(\overline{\mathbb{P}}-\chi_{\mathbf{d}} \otimes \chi_{\mathbf{d}}\right)-\frac{c_{1}\left(\mathbf{m}_{\mathbf{p}} \otimes \mathbf{m}_{\mathbf{p}}\right)-\chi_{\mathbf{m}} \otimes \chi_{\mathbf{m}}}{2 \sqrt{\Sigma_{l i m}}}
\end{aligned}
$$

where:

$$
\begin{gathered}
\chi_{\mathbf{d}}=\frac{\overline{\overline{\mathbb{P}}} \boldsymbol{\Sigma}_{\mathbf{p}}}{\sqrt{\overline{\overline{\mathbb{P}}} \boldsymbol{\Sigma}_{\mathbf{p}} \cdot \boldsymbol{\Sigma}_{\mathbf{p}}}} \\
\Sigma_{l i m}=c_{1}\left(\mathbf{m}_{\mathbf{p}} \otimes \mathbf{m}_{\mathbf{p}}\right) \boldsymbol{\Sigma}_{\mathbf{p}} \cdot \boldsymbol{\Sigma}_{\mathbf{p}}+c_{2} \boldsymbol{\Sigma}_{\mathbf{p}} \cdot \mathbf{m}_{\mathbf{p}}-R_{k}
\end{gathered}
$$




$$
\chi_{\mathbf{m}}=\frac{\left(2 c_{1} \boldsymbol{\Sigma}_{\mathbf{p}} \cdot \mathbf{m}_{\mathbf{p}}+c_{2}\right) \mathbf{m}_{\mathbf{p}}}{\sqrt{2 \Sigma_{\text {lim }}}}
$$

From Equations (27) and (29), one may conclude that $\chi_{\mathbf{d}}$ and $\mathbf{m}_{\mathbf{p}}$ are eigenvectors of the Hessian. The associated eigenvalues are respectively, $\Lambda_{1}=0$ and $\Lambda_{2}=\frac{3 \alpha_{d}^{4}}{2 \Sigma_{l i m}^{3 / 2}\left(3 \alpha_{m}^{2}-\left|\alpha_{d}^{2}\right|\right)}$, resulting in a positive semi-definite Hessian.

The eigenvalue $\Lambda_{2}$ is proved to be positive, but the null eigenvalue implies a singularity at any vector parallel to $\chi_{\mathrm{d}}$, which is at deviatory plane. Nevertheless, when Ulm-Gathier yield function tends to Drucker-Prager, $\Lambda_{2}$ tends also to null.

Since the Hessian of the hyperbolic function has at least one singularity, small perturbations are proposed in [5]. These perturbations are made along the eigenvector associated to the null eigenvalue.

\section{SEMI-ANALYTICAL LOWER BOUND SOLUTION}

Based on [12], a semi-analytical solution to scratch test is proposed. This method is developed under lower bound limit analysis theorem and the specimen is divided into three constant stress regions. The contact between the tool and the body is frictionless. Bard and Ulm [12] applied Mohr-Coulomb and Drucker-Prager on their solution; however, in this work the Ulm-Gathier yield function that considers porosity as a parameter is considered in this work.

On development of a lower bound solution the equilibrium requirements must be obeyed, such as: equilibrium with boundary conditions and equilibrium between adjacent regions. Figure 5 shows the partitioned specimen:

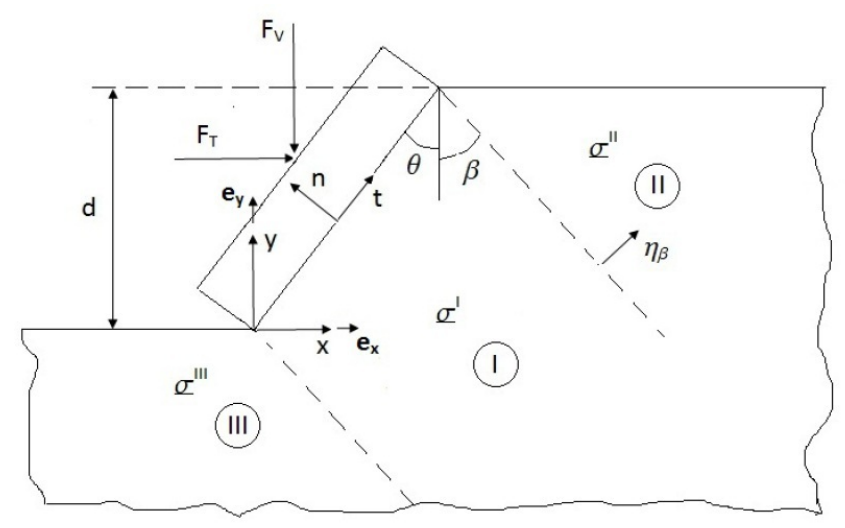

Figure 5. Partitioned specimen in three constant stress regions.

Since regions II and III have the same boundary conditions and the same normal angle between interfaces, only regions I and II will be analyzed. The interaction between indenter and the body is represented by an horizontal and a vertical forces, $F_{H}$ and $F_{V}$ respectively. The Cartesian axes $\{x, y\}$ are established as well as a local axes $\{t, n\}$. 


\subsection{Equilibrium}

From a region cut from region I, the equilibrium requirements are derived according to Figure 6:

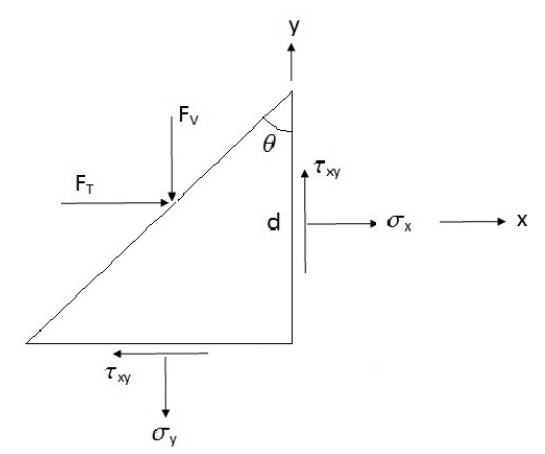

Figure 6. Exernal and internal forces equilibrium.

Considering a width $w$, the equilibrium is derived as in Equations 30 and 31:

1. $\sum F_{x}=0$

$$
\sigma_{x} w d-\tau_{x y} w d t g \theta+F_{T}=0 \Longrightarrow H_{T}=\tau_{x y} \operatorname{tg} \theta-\sigma_{x}
$$

2. $\sum F_{y}=0$

$$
-\sigma_{y} w d t g \theta+\tau_{x y} w d-F_{V}=0 \Longrightarrow H_{V}=\tau_{x y} \operatorname{cotg} \theta-\sigma_{y}
$$

onde: $H_{T}=F_{T} /(w d)$ e $H_{V}=F_{V} /(w d t g \theta)$.

Thus, the stress tensor in region I and the external loading are defined as in Equations 32 and 33 the equilibrium is written as in Equation 34.

$$
\begin{gathered}
\sigma^{I}=\sigma_{x}\left(\mathbf{e}_{\mathbf{x}} \otimes \mathbf{e}_{\mathbf{x}}\right)+\sigma_{y}\left(\mathbf{e}_{\mathbf{y}} \otimes \mathbf{e}_{\mathbf{y}}\right)+\tau_{x y}\left(\mathbf{e}_{\mathbf{x}} \otimes \mathbf{e}_{\mathbf{y}}\right)+\tau_{x y}\left(\mathbf{e}_{\mathbf{y}} \otimes \mathbf{e}_{\mathbf{x}}\right) \\
H=-H_{T}\left(\mathbf{e}_{\mathbf{x}} \otimes \mathbf{e}_{\mathbf{x}}\right)-H_{V}\left(\mathbf{e}_{\mathbf{y}} \otimes \mathbf{e}_{\mathbf{y}}\right) \\
\sigma^{I} \cdot \mathbf{n}=H \cdot \mathbf{n}
\end{gathered}
$$

When analyzing the boundary conditions at regions II and III, there is no external loads applied at these surfaces. It can be mathematically expressed as $\sigma^{I I} \cdot \mathbf{e}_{\mathbf{y}}=\sigma^{I I I} \cdot \mathbf{e}_{\mathbf{y}}=0$ and the stress tensors are written as:

$$
\sigma^{I I}=\sigma^{I I I}=\sigma_{x}\left(\mathbf{e}_{\mathbf{x}} \otimes \mathbf{e}_{\mathbf{x}}\right)
$$

Once defined these tensors, the equilibrium between interfaces I and II are established in Equation 36:

$$
\sigma^{I} \cdot \eta_{\beta}=\sigma^{I I} \cdot \eta_{\beta}
$$


On indenter-material interface, the tangential and normal stresses are written in local axes reference. Tangential and normal stresses are calculated as $\tau_{n t}=\mathbf{t} \cdot \sigma^{I} \cdot \mathbf{n}$ and $\sigma_{n}=$ $\mathbf{n} \cdot \sigma^{I} \cdot \mathbf{n}$, respectively. These components are also related to external loading, according to Equations 37 and 38:

$$
\begin{gathered}
\tau_{n t}=\frac{\sigma_{y}^{I}-\sigma_{x}^{I}}{2} \operatorname{sen}(2 \theta)-\tau_{x y} \cos (2 \theta)=\left(H_{T}-H_{V}\right) \cos \theta \operatorname{sen} \theta \\
\sigma_{n}=\sigma_{x}^{I} \cos ^{2} \theta+\sigma_{y}^{I} \operatorname{sen}^{2} \theta-\tau_{x y} \operatorname{sen}(2 \theta)=H_{T} \cos ^{2} \theta-H_{V} \operatorname{sen}^{2} \theta \leq 0
\end{gathered}
$$

As a remark, since contact between indenter and material must be provided, the condition $\sigma_{n} \leq 0$ must be met.

\subsection{Solution for a frictionless contact}

Since contact is frictionless, the shear component $\tau_{n t}=0$ and using Equation 37, $H_{T}=H_{V}$. Figure 7 shows the stress state at an infinitesimal element at region I:

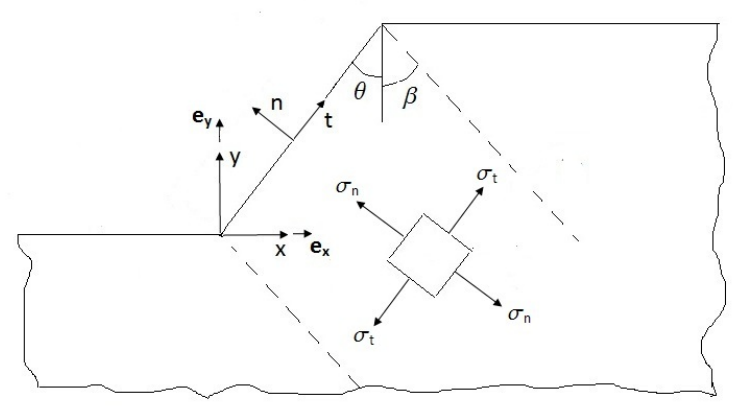

Figure 7. Stress state at region I.

It also can be observed that there are only normal stresses at region I and it can conclude that they are principal stresses and $\{\mathbf{t}, \mathbf{n}\}$ are eigenvectors of $\sigma^{I}$. On eigenvector basis:

$$
\sigma^{I}=\sigma_{t} \mathbf{t} \otimes \mathbf{t}+\sigma_{n} \mathbf{n} \otimes \mathbf{n}
$$

Studying the interface I-II and defining the normal vector $\eta_{\beta}=\operatorname{sen}(\theta+\beta) \mathbf{t}-\cos (\theta+$ $\beta) \mathbf{n}$, from equilibrium between these interfaces, given by $\sigma^{I} \cdot \eta_{\beta}=\sigma^{I I} \cdot \eta_{\beta}$, the stresses components of regions I, II and III are re-written according to Equations 40 and 41:

$$
\begin{aligned}
& \sigma^{I}=-\frac{H_{T} t g \theta}{\operatorname{tg}(\theta+\beta)}(\mathbf{t} \otimes \mathbf{t})-H_{T}(\mathbf{n} \otimes \mathbf{n}) \\
& \sigma^{I I}=\sigma^{I I I}=-H_{T}(1-\operatorname{tg} \theta \operatorname{tg} \beta)(\mathbf{t} \otimes \mathbf{t})
\end{aligned}
$$

In spite of not being represented in the stress tensors, the component $\sigma_{z}$ exists and it is calculated from plane strain hypothesis, in terms of others components $\sigma_{x}$ and $\sigma_{y}$ and dependent of the yield function. According to normality law: 


$$
\dot{\epsilon}_{z}=\dot{\lambda} \nabla f=0
$$

\subsection{The optimization problem}

Once the stress tensors are defined in Equations 40 and 41, the collapse factor is reached when regions I, II and III plasticizes. The optimization problem is stated as in Equation 43

$$
H_{s} \geq \max _{\beta} H_{T} \mid f\left(\sigma^{I}\right)=f\left(\sigma^{I I}\right)
$$

This procedure is called semi-analytical due to absence of a closed form solution. In order to solve this problem, it was developed a routine in MatLab(c)to find the angle $\beta$ that makes true the equality $f\left(\sigma^{I}\right)=f\left(\sigma^{I I}\right)$.

\section{RESULTS AND DISCUSSION}

\subsection{Validation}

Before applying the Ulm-Gathier considering porosity as a variable, it is important to compare the Limit Analysis and Finite Element model with those one existent in literature. Most models found such as [12] considers Mohr-Coulomb and Drucker-Prager yield functions on the solution.

As seen on previous section, when porosity tends to null, the Ulm-Gathier function tends to Drucker-Prager criterion and it is applied on finite element model. This approach is also applied to the semi-analytical solution.

Figure 8 shows the comparisons between Finite Element solution, semi-analytical solution and results from [12]. Some collapse factors are evaluated as long as friction angle varies:

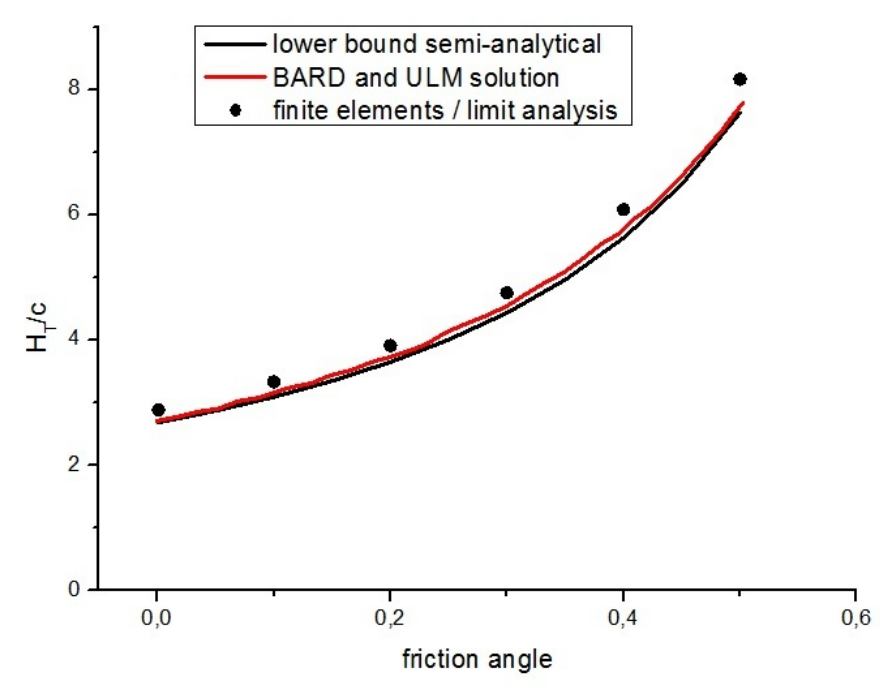

Figure 8. Collapse factors for many friction angles. 


\subsection{Porous materials results}

After model validation with Drucker-Prager yield function, the porosity effects are taken into account. At first, Figure 9 shows an example of an adaptive mesh.

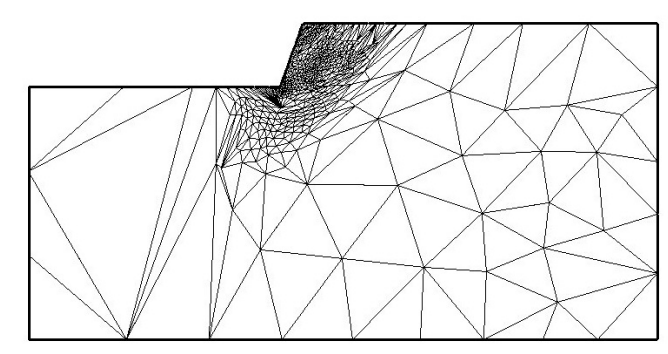

Figure 9. An adaptive mesh for the scratch test.

In order to evaluate the behavior of the collapse factors with porosity, some cases were studied considering a fixed friction angle and making the packing density to vary. The cases were run in the Limit Analysis Program and the results were compared to the ones from the semi-analytical solution. The friction angles considered were $\alpha_{s}=0.10\left(5.75^{\circ}\right)$ and $\alpha_{s}=0.80\left(64.4^{\circ}\right)$. Figures 10 and 11 shows the hardeness variation with packing density.
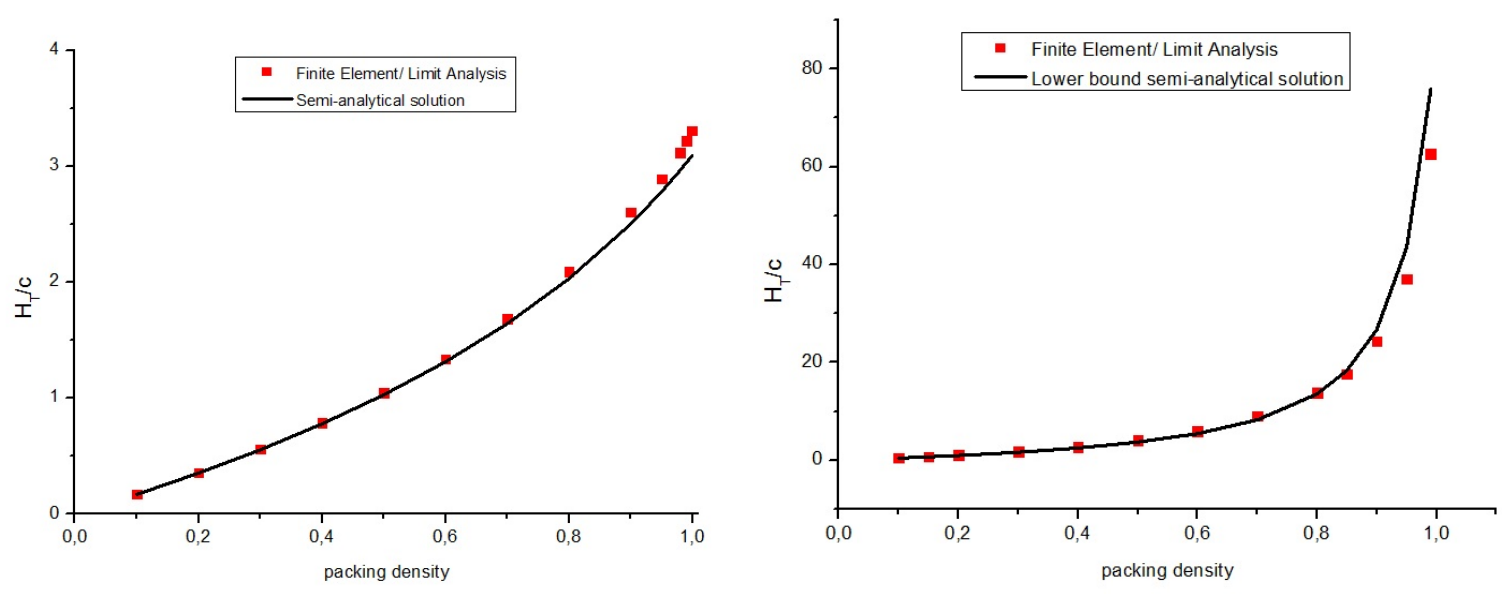

Figure 10. Results for friction angle $\alpha_{s}=0.10$. Figure 11. Results for friction angle $\alpha_{s}=0.80$. 


\subsection{Discussion}

The scratch test is an old method to determine material mechanical properties and this technique has gained interest due to the properties implied during test, such as adherence, hardness, cohesion and elasticity. In this problem, a rigid indenter is dragged on a specimen at a constant depth and it can be modeled as a plasticity problem. The problems in plasticity may be solved by an incremental method or by direct method like limit analysis. The main advantage of limit analysis method is the absence of stress calculation at each load step and the main interest is the determination of the load factor that multiplies a reference load that will cause the incipient plastic collapse. The basic statement of limit analysis is found at most known literature in plasticity theory. These concepts were extended to mathematical programming and limit analysis problems are solved by an optimization problem with restrains.

In order to analyze the behavior in plasticity of porous material, a suitable yield function must be used and it was seen that classical functions used such Mohr-Coulomb or Drucker-Prager do not consider porosity effects. The Ulm-Gathier function applied and implement in the Limit Analysis Program considers porosity effects throughout Mori-Tanaka morpholy. This function was implemented in Limit Analysis Program and the eigenvalues and eigenvectors of the Hessian shall be analyzed. If Ulm-Gathier function is elliptical, the positive-definiteness of the Hessian can be proved. Otherwise, if the function has a hyperbolical shape, an alternative development for plane strain is made and the Hessian is positive semi-definite. This Hessian characteristic shall be studied since it has to be invertible in order to apply the limit analysis solver algorithm, based on a quasi-Newton method. So as to solve that, a small perturbation along the eigenvector associated to null eigenvalue is made.

After computational implementation of the Ulm-Gathier function, some cases considering Drucker-Prager criterion were analyzed in order to validate the model and compare the results to these ones existing in literature. A lower-bound semi-analytical solution was also developed. The graph in Figure 8 shows that the semi-analytical solutions are very close to the Finite Element model.

Once validated the model, some cases considering porosity effects were studied. Qualitatively analyzing the variation of the collapse factors along packing density, one may observe it increases when porosity diminishes. When $\eta \rightarrow 1$, the Ulm-Gathier function tends to Drucker-Prager results at the friction angles in Figure 8.

As known, the contact between material and tool is not frictionless. However, to model and implement friction models is a difficult task and it is the next step. The work of [13] shows some contact laws and it will be studied in order to implement in analysis limit problems. Also, [14] proposed a formulation for friction problems based on [15]. However, it is limited to some friction coefficients and some specific applications.

Furthermore, a 3-D model shall be developed, since in scratch test problems there is no symmetric and the specimen has width larger than the tool. 


\section{REFERENCES}

\section{References}

[1] R. Bard and F. J. Ulm, "An analysis of scratch tests on porous cohesive-frictional material," 2009.

[2] B. Gathier, Multi Scale Homogenization - Application to Shale Nanoindentation. PhD thesis, Massachussetts Institute of Technology, Massachussetts, 2006.

[3] S. Cariou, The Effect of the Packing Density on the Indentation Hardness of CohesiveFrictional Porous Materials. PhD thesis, Massachussetts Institute of Technology, Massachussetts, 2006.

[4] N. Zouain, J. Herskovits, L. Borges, and R. Feijo, "An interative algorithm for limit analysis with nonlinear yield functions," International Journal of Solids and Structures, vol. 30, pp. 1397-1417, 1993.

[5] I. D. S. Pontes, L. Borges, N. Zouain, and F. Lopes, "An approach to limit analysis with cone-shaped yield surfaces," International Journal for Numerical Methods in Engineering, vol. 40, pp. 4011-4032, 1997.

[6] W. F. Chen and X. L. Liu, Limit Analysis in Soil Mechanics. Amsterdam: Elsevier Science Publishing, 1990.

[7] W. F. Chen and X. L. Liu, Limit Analysis and Soil Plasticity. Amsterdam: Elsevier Science Publishing, 1975.

[8] J. Lubliner, Plasticity Theory. USA: Pearson Education, 1990.

[9] L. M. Kachanov, Foundations of the Teory of Plasticity. Netherlands: North-Holland Publishing Company, 1971.

[10] J. Silveira, "Elementos finitos triangulares mistos para anlise limite em estado plano," Master's thesis, Universidade Federal do Rio de Janeiro, Rio de Janeiro, 1991.

[11] L. Borges, N. Zouain, C. Costa, and R. Feijo, "An adaptative approach to limit analysis," International Journal of Solids and Structures, vol. 38, pp. 1707-1720, 2001.

[12] R. Bard and F. J. Ulm, "Scratch hardness-strength solutions for cohesive-frictional materials," International Journal for Numerical and Analytical Methods in Geomechanics, 2011.

[13] Z. Q. Feng, M. Hjiaj, G. Saxc, and Z. Mrz, "Effect of frictional anisotropy on the quasistatic motion of a deformable solid sliding on a planar surface," Computational Mechanics, vol. 37, pp. 349-361, 2006.

[14] L. Naccarato, Anlise Limite com Condies de Contorno Unilaterais. PhD thesis, Universidade Federal do Rio de Janeiro, Rio de Janeiro, 2006. 
[15] G. Saxc and L. Boussine, "Limit analysis theorems for implicit standard materials: Application to the unilateral contact with dry friction and the non-associated flow rules in soils and rocks," International Journal of Mechanical Science, vol. 40, pp. 387-398, 1998. 